\title{
Le syndrome postgarde de nuit chez les médecins urgentistes : caractéristiques et facteurs influençants
}

\section{Post-shift syndrome among the emergency physicians: characteristics and factors of influence}

\author{
C. Fasula $\cdot$ A. Marchal $\cdot$ H. Krebs $\cdot$ C. Moser $\cdot$ R. Genre-Grandpierre $\cdot$ X. Bobbia $\cdot$ J.-E. de La Coussaye $\cdot$ \\ P.-G. Claret
}

Reçu le 18 décembre 2017; accepté le 27 février 2018

(C) SFMU et Lavoisier SAS 2018

Résumé Introduction : Les urgentistes connaissent une privation de sommeil en lendemain de garde. Beaucoup ont des perceptions ou des comportements inhabituels que nous appelons syndrome post-garde de nuit (PGN). L'objectif principal était de caractériser le syndrome PGN des urgentistes. Les objectifs secondaires étaient d'établir une note évaluant le syndrome PGN et de déterminer les facteurs influençant ce syndrome PGN.

Méthode : Étude prospective de cohorte, observationnelle, multicentrique, auprès des urgentistes du Gard et de l'Hérault entre janvier et juin 2017. Deux questionnaires informatiques anonymes étaient diffusés aux structures d'urgences du Gard et de l'Hérault. En journée normale, hors sortie de garde, un questionnaire colligeait les symptômes ressentis habituellement en lendemain de garde, à coter entre 0 et 10 . En sortie de garde, un autre questionnaire s'intéressait au ressenti de la garde réalisée et aux caractéristiques de cette garde. Les médecins étaient encouragés à répondre aux deux questionnaires.

Résultats : Cent treize médecins (45\%) ont répondu, 67 (59\%) à distance d'une garde et $46(41 \%)$ en post-garde immédiat. Le syndrome PGN comprend des symptômes somatiques (échelle visuelle analogique $(\mathrm{EVA})=5[2-8]$ ), comportementaux $(\mathrm{EVA}=7$ [3-8]), liés à l'humeur $(\mathrm{EVA}=$ 7 [5-8]) et psychiques (EVA $=6$ [4-8]). Ces symptômes en post-garde immédiat étaient ressentis de manière moins

C. Fasula $(\bowtie) \cdot$ A. Marchal $\cdot$ H. Krebs $\cdot$ C. Moser .

R. Genre-Grandpierre $\cdot$ X. Bobbia $\cdot$ J.-E. de La Coussaye

P.-G. Claret

Pôle anesthésie-réanimation-douleur-urgences,

CHU de Nîmes, place du Professeur-Robert-Debré,

F-30029 Nîmes cedex 09, France

e-mail : cyrielle.fasula@gmail.com

J.-E. de La Coussaye

Université Montpellier-Nîmes, 2, rue École-de-Médecine,

F-34060 Montpellier, France importante que lorsque décrit à distance (respectivement $p=0,001, p<0,001, p<0,001, p=0,002)$. Les gardes de SMUR (service mobile d'urgence et de réanimation) et de traumatologie étaient associées à un syndrome PGN moins marqué (respectivement $p=0,035$ et $p=0,02$ ) que les gardes des filières médico-chirurgicales.

Conclusion : Il s'agit de la première évaluation du syndrome PGN chez les urgentistes français. Les symptômes en sont moins importants en post-garde immédiat. Les gardes de traumatologie et de SMUR semblent mieux tolérées.

Mots clés Médecine d'urgence · Syndrome post-garde de nuit $\cdot$ Privation de sommeil

Abstract Introduction: Emergency physicians suffer from sleep deprivation after being on shift. Lots have unappropriated behaviors called post-shift syndrome. This study aims to characterise the post-shift syndrome firstly and secondly to establish a note and factors of influence.

Methods: It is a prospective, cohort, observational, multicentric study in emergency units of Hérault and Gard between January and June 2017. A computerized and anonymized questionnaire was spread in these emergency units. During a normal day, a questionnaire compiled the symptoms evaluated between 0 and 10 . This defined the post-shift syndrome. After a night shift, another quiz gathered the shift feeling, the day after shift feeling, and data about the shift. Practitioners were encouraged to answer twice.

Results: One hundred and thirteen (45\%) emergency physicians answered, 67 (59\%) during a normal day versus 46 (41\%) after being on shift. The post-shift syndrome includes somatic disorders (visual analog scale $(\mathrm{VAS})=5[2-8]$ ), behavioral disorders (VAS $=7$ [3-8]), mood disorders (VAS $=7$ [5-8]), and mental health disorders (VAS $=6$ [4-8]). Immediate post-shift syndrome was less important (respectively $P=0.001, P<0.001, P<0.001$, and $P=0.002)$. Creation of a note for post-shift syndrome shows that SAMU and 
traumatology shifts are better endured (respectively $P=0.035$ and $P=0.02$ ).

Conclusion: This is the first evaluation of the post-shift syndrome. Symptoms are less important after being on shift. Traumatology and SAMU shifts are better stood.

Keywords Emergency medicine $\cdot$ Post-shift syndrome · Sleep deprivation

\section{Introduction}

Les médecins urgentistes sont confrontés à des événements inattendus, graves et souvent brutaux mettant la vie des gens en jeu. Ils doivent aussi gérer un flux de patients en constante augmentation et l'organisation d'une équipe médicale et paramédicale [1]. La médecine d'urgence se caractérise par un stress professionnel majeur, des prises de décisions importantes et des horaires de travail variables, souvent nocturnes $[2,3]$. Le syndrome d'épuisement professionnel des praticiens hospitaliers est favorisé par la pression quantitative, les conflits entre les organisations professionnelles ou personnelles, et la qualité du travail d'équipe [2,4]. Certains médecins urgentistes vont jusqu'à la reconversion professionnelle du fait des conditions de travail et des horaires décalés [5]. Selon l'étude SESMAT (santé et satisfaction des médecins au travail), $21 \%$ des médecins urgentistes ont l'intention de quitter leur profession contre $17 \%$ dans les autres spécialités médicales [6]. Les lendemains de garde sont marqués par un état de fatigue majeur. La privation répétée de sommeil et ses conséquences existent, mais sont variables d'un individu à l'autre [7]. En lendemain de garde, de nombreux médecins urgentistes reconnaissent avoir des perceptions, des comportements et des attitudes inhabituels en lien avec cette carence en sommeil. C'est ce qui est communément appelé le syndrome post-garde de nuit (PGN) [8]. Désigné ainsi, en admettant sa réalité, ce concept flou est peu décrit dans la littérature. Les objectifs de notre étude sont de caractériser le syndrome PGN des urgentistes et d'en déterminer les facteurs d'influence.

\section{Méthodes}

Étude prospective de cohorte, observationnelle, multicentrique, réalisée auprès des médecins urgentistes du Gard et de l'Hérault entre janvier et juin 2017. Ont été inclus les médecins urgentistes en activité du Gard et de l'Hérault (250 docteurs en médecine). Ont été exclus les praticiens non thésés, intérimaires ou remplaçants. L'objectif principal était de caractériser le syndrome PGN des urgentistes. Les objectifs secondaires étaient d'établir une note évaluant le syndrome PGN et de déterminer les facteurs influençant ce syndrome PGN. Le protocole a été approuvé par le comité d'éthique institutionnel du CHU de Nîmes. Avec accord de celui-ci et dans la mesure où les questionnaires étaient totalement anonymes, il n'y a pas eu nécessité de soumission à la CNIL. Deux questionnaires informatiques (Annexe A) étaient diffusés dans les structures d'urgences du Gard et de l'Hérault, après accord du chef de service pour le relayer à ses équipes. Ils ne comportaient pas de données permettant d'identifier le médecin interrogé. Ils étaient accompagnés d'un texte explicatif de l'étude et d'information sur l'utilisation des données. Si le médecin interrogé sortait de garde, il était dirigé vers un premier questionnaire (groupe post-garde immédiat) (Questionnaire A). Le médecin interrogé devait coter les symptômes qu'il avait ou non sur une échelle allant de 0 à 10 . Le ressenti de la garde était recensé. Les données objectives de la garde étaient colligées (nombre de passages, type de garde, durée de la garde, type d'établissement, nombre de places disponibles d'hospitalisation, nombre de gardes dans le mois, durée de la garde). Si le médecin ne sortait pas de garde, il était dirigé vers un second questionnaire (groupe à distance de garde) (Questionnaire B). Ce questionnaire portait sur les caractéristiques habituelles et globales de lendemain de garde. Cela permettait de définir le syndrome PGN dans sa globalité. Tous les praticiens interrogés avaient une activité mixte, intra et extrahospitalière. Les médecins étaient encouragés à répondre deux fois à ces questions, une fois le lendemain d'une garde (questionnaire du groupe post-garde immédiat) et une fois lors d'une journée normale (questionnaire du groupe à distance). Les symptômes à coter dans notre étude étaient issus d'une synthèse de questionnaires (Maslach Burnout Inventory) et des caractéristiques les plus courantes de la privation de sommeil $[8,9]$. Nous avons utilisé l'échelle visuelle analogique (EVA) pour coter les différents symptômes ressentis car l'EVA (échelle visuelle analogique) est moins sensible au biais de confusion, qu'elle est rapide et simple à réaliser tout en étant facilement reproductible [10]. Pour pouvoir évaluer, comparer et analyser les caractéristiques du syndrome PGN, nous avons choisi d'établir une note. Les EVA des différentes catégories (somatique, comportement, humeur, psyché) des questionnaires réalisés en post-garde ont été moyennées et ramenées à une note sur 200. Plus la note était basse, meilleur (ou moins marqué) était le syndrome PGN.

\section{Analyse statistique}

Les résultats statistiques étaient présentés sous forme de médianes et d'écarts interquartiles pour les variables quantitatives. Pour les variables qualitatives, les effectifs et les pourcentages associés étaient présentés. Les variables qualitatives étaient comparées par un test du $\mathrm{Chi}^{2}$. À défaut, si les conditions de réalisation de ce test n'étaient pas respectées, le test exact de Fisher était utilisé. Les variables quantitatives 
étaient comparées entre les groupes par un test $t$ de Student. Les facteurs explicatifs potentiels (facteurs de risques ou facteurs protecteurs) de présenter un syndrome PGN positif ou négatif étaient analysés en univarié puis en multivarié à l'aide d'un modèle de régression logistique. Les variables candidates pour le modèle multivarié étaient les variables présentant une association avec valeur de $p<0,2$ en analyse univariée. Le risque d'erreur de première espèce était fixé à $5 \%$. Les données étaient, initialement, colligées sur Microsoft Excel ${ }^{\circledR}$ (Microsoft Corporation, Redmond, États-Unis). L'analyse statistique était réalisée à l'aide du logiciel de statistique R version 3.2.2 (R Foundation for Statistical Computing, Vienne, Autriche).

\section{Résultats}

Au total, 113/250 médecins (45\%) ont répondu au questionnaire : $67(59 \%)$ dans le groupe à distance versus $46(41 \%)$ dans le groupe post-garde immédiat. L'âge médian était de 33 ans [intervalle interquartile : $30-41$ ]. Le ratio hommes/ femmes était de 0,95. Les principales données démographiques sont présentées dans le tableau 1. Il n'y a pas de différence significative entre les deux groupes de population. Pour les questionnaires en post-garde immédiat, les caractéristiques des gardes ont été également recensées. Elles sont présentées dans le tableau 2.

Les médecins urgentistes, interrogés en extra clinique, décrivent des symptômes cotés entre 0 et 10 et présentés dans les tableaux 3, 4. Ils déclarent consommer du café en post-garde pour $28 \%$ d'entre eux et du tabac pour $9 \%$. En revanche, ils ne consomment pas de substances psychoactives en lendemain de garde. En interrogeant les médecins à distance d'une garde ou en post-garde immédiat, on note des différences significatives concernant les symptômes somatiques $(p=0,001)$, les symptômes comportementaux

\begin{tabular}{|ll|}
\hline Tableau 2 Caractéristiques des gardes & \\
\hline Variables & $\boldsymbol{n}=\mathbf{4 6}$ \\
\hline Type de garde & \\
Régulation & $5(11 \%)$ \\
SMUR & $15(33 \%)$ \\
Déchocage & $11(24 \%)$ \\
Filière médico-chirurgicale & $27(59 \%)$ \\
Traumatologie & $8(17 \%)$ \\
Pédiatrie & $5(11 \%)$ \\
Durée de la garde & \\
24 heures & $39(85 \%)$ \\
12 heures & $7(15 \%)$ \\
Type d'établissement & \\
CHU & $36(78 \%)$ \\
CHR & $10(22 \%)$ \\
Nombre de passage & \\
Moins que la moyenne & $2(4 \%)$ \\
Dans la moyenne & $35(78 \%)$ \\
Plus que la moyenne & $8(18 \%)$ \\
Détails concernant la garde & \\
Nombre d'heures de sommeil & $2,0[1,0-3,7]$ \\
Nombre de gardes dans le mois & $4,5[4,0-6,7]$ \\
Complexité des pathologies rencontrées & $6,0[4,0-6,0]$ \\
Accessibilité des examens & $4,5[3,0-6,7]$ \\
complémentaires & \\
Qualité du travail d'équipe & $4,0[3,0-6,0]$ \\
Accessibilité des avis spécialisés & $4,0[3,0-6,0]$ \\
Efficacité de l'interne & $5,0[3,5-7,0]$ \\
\hline CHR : centre hospitalier régional ; CHU : centre hospitalier uni- \\
versitaire; SMUR : service mobile d'urgence et de réanimation. \\
Résultats exprimés en médianes (interquartile) et valeurs abso- \\
lues (pourcentages) \\
\end{tabular}

\begin{tabular}{|lllll|}
\hline \multicolumn{2}{|l|}{ Tableau 1 Caractéristiques de la population } & & \\
\hline Variables & $\begin{array}{l}\text { Population totale } \\
(\boldsymbol{n}=\mathbf{1 1 3})\end{array}$ & $\begin{array}{l}\text { Population interrogée } \\
\text { à distance }(\boldsymbol{n}=\mathbf{6 7})\end{array}$ & $\begin{array}{l}\text { Population post-garde } \\
\text { immédiat }(\boldsymbol{n}=\mathbf{4 6})\end{array}$ & Valeur de P \\
\hline Âge (ans) & $33[30-41]$ & $33[30-44]$ & $33[31-38]$ & 1 \\
Femmes & $58(51 \%)$ & $34(52 \%)$ & $24(51 \%)$ & 1 \\
Ancienneté aux urgences & $6,0[3,0-12,0]$ & $5,5[3,0-13,7]$ & $6,0[2,0-9,0]$ & 0,429 \\
$($ ans) & & & $7(15 \%)$ & 1 \\
Célibataire & $16(14 \%)$ & $9(14 \%)$ & $23(49 \%)$ & 0,473 \\
En couple & $61(54 \%)$ & $38(58 \%)$ & $16(34 \%)$ & 0,353 \\
Marié/e & $32(28 \%)$ & $16(24 \%)$ & $1(2 \%)$ & 0,866 \\
Divorcé/e & $4(4 \%)$ & $3(4 \%)$ & $24(51 \%)$ & 1 \\
Enfants & $58(51 \%)$ & $34(51 \%)$ & & \\
\hline Résultats exprimés en médianes (interquartile) et valeurs absolues (pourcentages) & & \\
\hline
\end{tabular}




\begin{tabular}{|c|c|c|c|}
\hline Variables & $\begin{array}{l}\text { Population } \\
\text { interrogée } \\
\text { à distance } \\
(n=67)\end{array}$ & $\begin{array}{l}\text { Population } \\
\text { post-garde } \\
\text { Immédiat } \\
(n=46)\end{array}$ & $\begin{array}{l}\text { Valeur } \\
\text { de } p\end{array}$ \\
\hline \multicolumn{4}{|l|}{$\begin{array}{l}\text { Symptômes } \\
\text { somatiques, } \\
\text { médiane }\end{array}$} \\
\hline Fatigue & 8 [7-9] & $7[6-8]$ & \\
\hline Douleurs diffuses & $5[3-7]$ & $4[1,25-6]$ & \\
\hline $\begin{array}{l}\text { Sensations } \\
\text { de malaise }\end{array}$ & $2[0-6]$ & $1,5[0-5]$ & \\
\hline Gastralgies & $1[0-5]$ & $0[0-2]$ & \\
\hline Céphalées & $6[2-8]$ & $3[0-7]$ & \\
\hline Moyenne & $5[2-8]$ & $4[0-7]$ & 0,001 \\
\hline \multicolumn{4}{|l|}{$\begin{array}{l}\text { Symptômes } \\
\text { comportementaux, } \\
\text { médiane }\end{array}$} \\
\hline $\begin{array}{l}\text { Achats } \\
\text { inconsidérés }\end{array}$ & $7[3-8]$ & $0[0-5]$ & \\
\hline Cynisme & $7[4-8]$ & $5[1,25-6]$ & \\
\hline Actes inachevés & $7[4-7,5]$ & $5,5[3-7]$ & \\
\hline $\begin{array}{l}\text { Engagements } \\
\text { excessifs }\end{array}$ & $3[0-7]$ & $1[0-5]$ & \\
\hline $\begin{array}{l}\text { Troubles } \\
\text { de la fluence } \\
\text { verbale }\end{array}$ & $7[5-8]$ & $5[5-7]$ & \\
\hline Moyenne & $7[3-8]$ & $5[0-6]$ & $<0,001$ \\
\hline \multicolumn{4}{|l|}{$\begin{array}{l}\text { Troubles } \\
\text { de l'humeur, } \\
\text { médiane }\end{array}$} \\
\hline Irritabilité & $8[7-9,5]$ & $7[6-8]$ & \\
\hline Sautes d'humeur & 7 [6-9] & $6,5[4,25-8]$ & \\
\hline Impulsivité & $7[5-8]$ & $6[3,25-7]$ & \\
\hline Anxiété & $7[4-8]$ & $5[2-6]$ & \\
\hline Intolérance & $7[6-8]$ & $5[3-7]$ & \\
\hline \multicolumn{4}{|l|}{$\begin{array}{l}\text { Symptômes } \\
\text { psychiques, } \\
\text { médiane }\end{array}$} \\
\hline $\begin{array}{l}\text { Ralentissement } \\
\text { des idées }\end{array}$ & $7[5-8]$ & $7[4-8]$ & \\
\hline Manque du mot & $7[4-8]$ & $6[3,25-7]$ & \\
\hline $\begin{array}{l}\text { Sentiment d'être } \\
\text { influençable }\end{array}$ & $5[1-6]$ & $5[1-6]$ & \\
\hline Oublis fréquents & $6[5-8]$ & $5[2,25-7]$ & \\
\hline $\begin{array}{l}\text { Troubles } \\
\text { de l'attention }\end{array}$ & $7[5-8]$ & $6[4,25-7,75]$ & \\
\hline Moyenne & $6[4-8]$ & $6[3-7]$ & 0,002 \\
\hline
\end{tabular}

Résultats exprimés en médianes (interquartile) et valeurs absolues (pourcentages)
Tableau 4 Caractéristiques des consommations en syndrome post-garde de nuit

\begin{tabular}{|c|c|c|c|}
\hline Variables & $\begin{array}{l}\text { Population } \\
\text { interrogée } \\
\text { à distance }\end{array}$ & $\begin{array}{l}\text { Population } \\
\text { post-garde } \\
\text { immédiat }\end{array}$ & $\begin{array}{l}\text { Valeur } \\
\text { de } p\end{array}$ \\
\hline \multicolumn{4}{|c|}{ Consommations, $n(\%)$} \\
\hline Cafés & $32(28,3 \%)$ & $25(22,1 \%)$ & 0,49 \\
\hline Tabac & $10(8,8 \%)$ & $9(8 \%)$ & 0,52 \\
\hline Cannabis & $1(0,9 \%)$ & $1(0,9 \%)$ & 0,80 \\
\hline $\begin{array}{l}\text { Médicaments } \\
\text { hypnotiques }\end{array}$ & $3(2,7 \%)$ & $3(2,7 \%)$ & 0,64 \\
\hline $\begin{array}{l}\text { Médicaments } \\
\text { stimulants }\end{array}$ & $0(0 \%)$ & $0(0 \%)$ & 1 \\
\hline \multicolumn{4}{|c|}{ Durée des signes, $n$ (\%) } \\
\hline Matinée & $1(0,9 \%)$ & $0(0 \%)$ & 0,42 \\
\hline Après-midi & $4(3,5 \%)$ & $4(3,5 \%)$ & 0,58 \\
\hline Soirée & $12(10,6 \%)$ & $7(6,2 \%)$ & 0,71 \\
\hline Le lendemain & $49(43,4 \%)$ & $35(31 \%)$ & 0,73 \\
\hline $\begin{array}{l}\text { Résultats exprin } \\
\text { lues (pourcentag }\end{array}$ & Ines (inte & rtile) et val & urs abso- \\
\hline
\end{tabular}

$(p<0,001)$, les troubles de l'humeur $(p<0,001)$ et les symptômes psychiques $(p=0,002)$ (Fig. 1 et Tableau 3 ). Les symptômes sont moins importants lorsqu'ils sont décrits en lendemain de garde que lorsqu'ils sont décrits à distance. La note médiane du syndrome PGN était de 89 [73,5-157,5]. En analyse bivariée, les caractéristiques individuelles n'influencent pas sur la note. Les gardes de SMUR (service mobile d'urgence et de réanimation) et de traumatologie sont associées des syndromes PGN moins importants (respectivement $p=0,035$ et $p=0,02$ ) que celles en filière médico-chirurgicale (Fig. 2). En analyse multivariée, ces variables restent indépendantes après ajustement sur la durée de la garde ( 24 vs 12 h), le nombre d'heures de sommeil durant la garde et le nombre de gardes dans le mois de la réponse au questionnaire.

\section{Discussion}

Interroger les médecins urgentistes de l'Hérault et du Gard a permis de caractériser le syndrome PGN. La cohorte de signes comprend des troubles somatiques à 5/10 [2-8], des troubles comportementaux à $7 / 10$ [3-8], des troubles de l'humeur à 7/10 [5-8] et des symptômes psychiques à 6/10 [4-8]. Il existe une différence entre l'évaluation à distance et en post-garde immédiat. Les médecins ont une note de syndrome PGN immédiat plus basse que lorsqu'ils l'évaluent à distance. Les gardes en filière médico-chirurgicale entrainent les syndromes PGN plus sévères que les gardes 


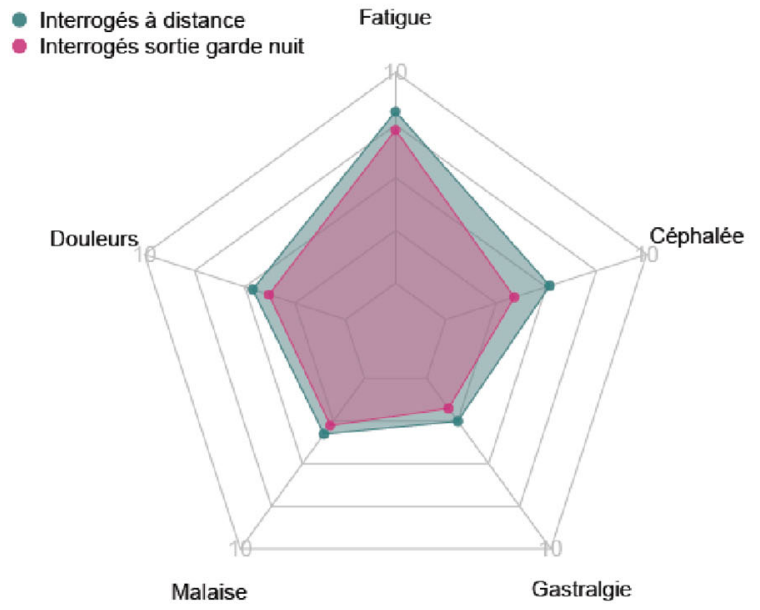

A

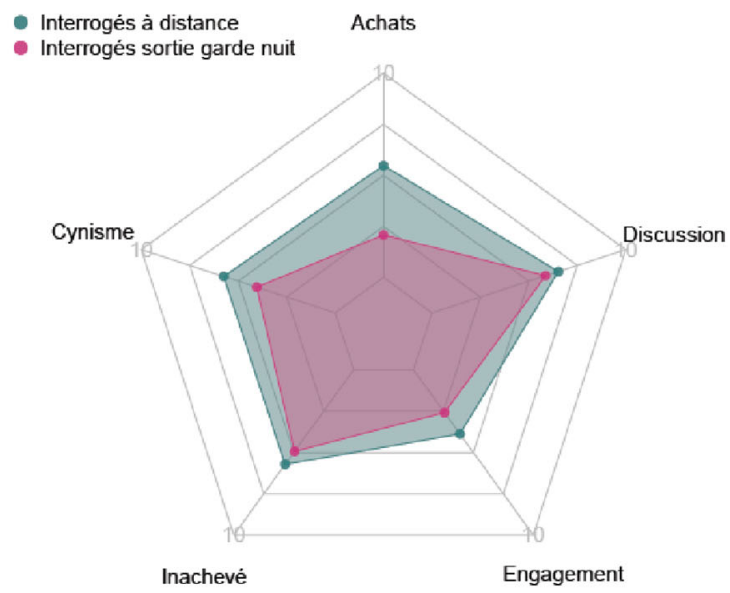

B

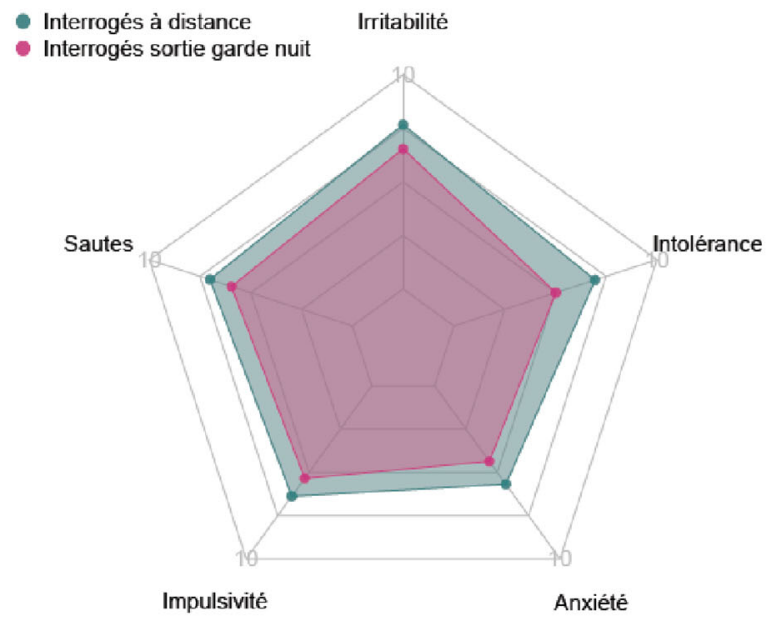

C

D

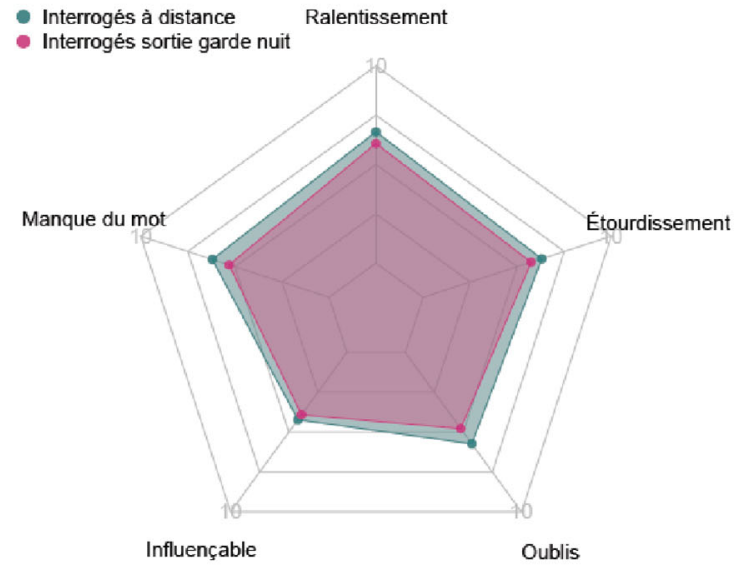

Fig. 1 Comparaison des syndromes post-garde de nuit entre les populations interrogées à distance d'une garde et en post-garde immédiat. A. Symptômes somatiques ; B. Symptômes comportementaux ; C. Troubles de l'humeur ; D. Symptômes psychiques

en traumatologie ou en SMUR. Aussi, la question de la réalité immédiate de ce syndrome se pose. L'évaluation à distance des repos de garde montre qu'il s'agit d'une appréciation plus générale des repos de garde qui en ferait un syndrome existant. Notre étude comporte des limites. En effet, les structures d'urgences dans lesquelles a été menée cette enquête ont des sectorisations et des recours techniques différents. Ces résultats peuvent aussi être influencés par un biais de sélection. La garde réalisée peut influencer les médecins dans leur envie ou leur démarche pour répondre. Ceci pourrait expliquer un taux de réponse de $45 \%$ et non plus. Aussi, il existe un effet Hawthorne lié au fait que les sujets ont conscience de participer à une expérience dans laquelle ils sont testés, il peut s'agir d'un biais de notre étude. Selon Fido et al., les travailleurs de nuit (tous métiers confondus) subissent plus de stress professionnel, ont un niveau de performance plus faible, ont une capacité de concentration plus basse, font plus d'erreurs et ont plus d'accidents du travail que les employés de jour [11]. La privation expérimentale d'une nuit de sommeil conduit à un raisonnement rigide, à des erreurs répétées, à des difficultés à apprécier une nouvelle situation et surtout à des troubles de l'humeur [12-14]. Ces éléments peuvent expliquer une évaluation moins importante du syndrome PGN immédiat par rapport à l'évaluation à distance. Dans la littérature, la comparaison des capacités des chirurgiens avant et après une garde montre des erreurs de pensée, des troubles de la mémoire et des troubles de l'attention, mais sans 


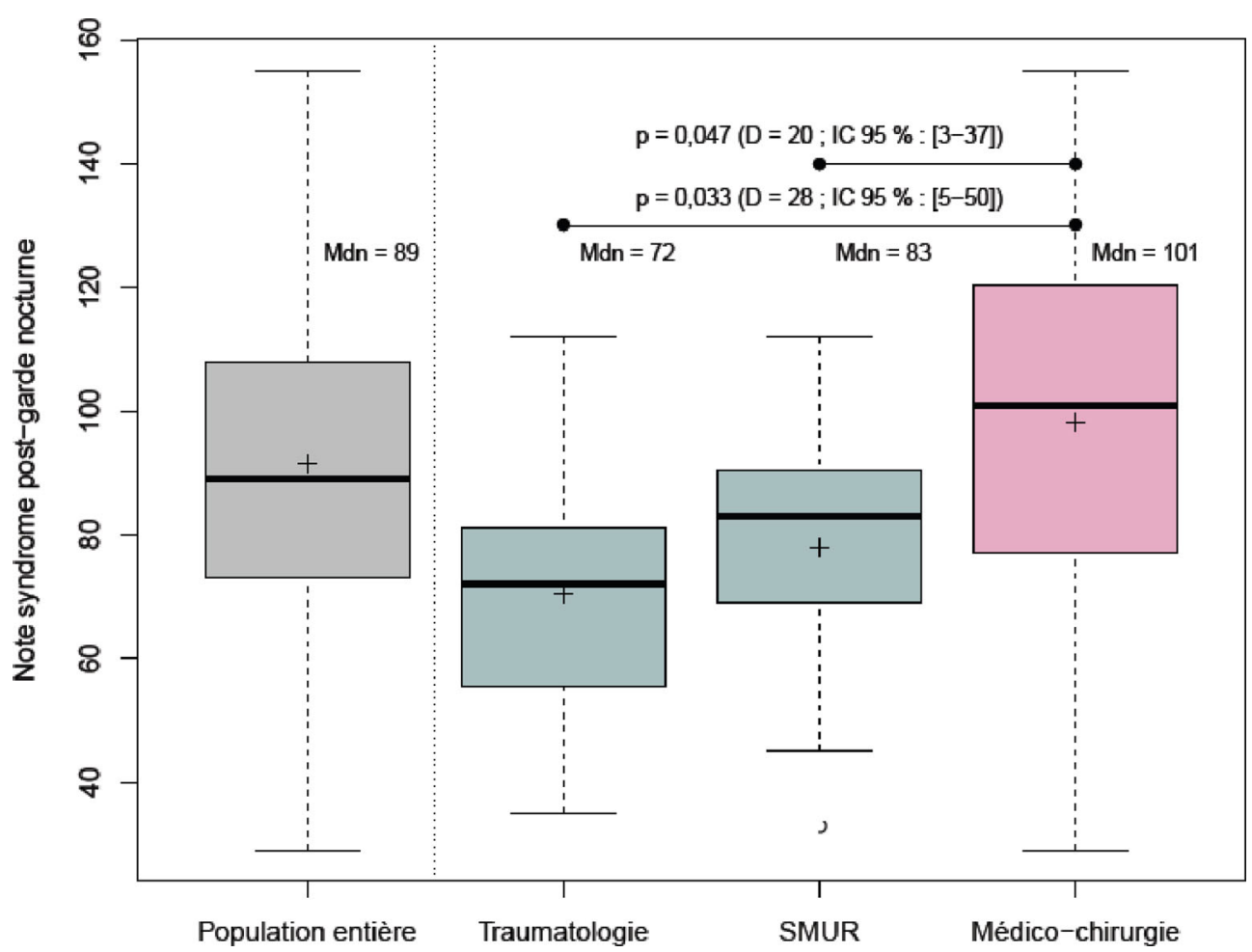

Fig. 2 Comparaison des syndromes post-garde de nuit en fonction du type de garde. Mdn : médiane ; $p:$ valeur de $p$

conséquence pour le patient et sa santé [15]. Une étude récente de Maric et al. a cherché le lien entre la privation de sommeil et ces comportements aberrants. Elle met en évidence une modification des ondes lentes du sommeil (enregistrées lors d'électroencéphalogrammes (EEG) de repos) qui pourrait expliquer ces symptômes [16]. En effet, la privation de sommeil entraine des changements de métabolisme cérébral et de l'activation neuronale [17-19]. Chez les travailleurs de nuit, toutes professions confondues, la consommation de tabac, de thé, de café est plus importante que chez les employés de jour [20]. Selon Ker et al., la caféine améliore le raisonnement et la conceptualisation, la mémoire, l'orientation, l'attention et la perception. Inversement, elle n'a pas de bénéfice sur le fonctionnement verbal et les compétences linguistiques [21]. À l'inverse de la privation de sommeil, la caféine diminue les prises de risque [22]. Elle améliore également les EEG enregistrés après un manque de sommeil. On comprend donc pourquoi les médecins urgentistes boivent plus de cafés en repos de garde pour se tenir éveillés ou pour conduire $[23,24]$. Néanmoins, notre étude ne montre pas une majoration de la consommation de tabac par rapport à la consommation moyenne des Français. Le manque de sommeil induit des troubles de concentration, de mémoire, de réflexion, d'interprétation et des troubles émotionnels [11,15]. Il semble donc logique d'avoir une évaluation éloignée de la réalité de son propre état en lendemain de garde comme retrouvé dans la littérature [25].
Dans notre travail, l'âge, l'ancienneté aux urgences ou le sexe ne semblent pas influencer significativement la note de syndrome PGN. En 2001, Reid et al. met en évidence que les plus jeunes ont une meilleure résistante à la privation de sommeil que les plus âgés [26]. Les femmes et les hommes déploient des stratégies de gestion des situations de fatigue et de stress différentes. Les femmes du stress ont des gestions plus négatives avec évitement, fuites, ruminations, résignations [1]. Bae et al. rapporte aussi des différences hommesfemmes : désorganisation du sommeil pour les hommes, troubles du sommeil et consommation de tabac plus importante pour les femmes [27]. Ces éléments de la littérature semblent être en désaccord avec nos résultats. Les notes liées aux gardes de SMUR et de traumatologie sont significativement plus basses. À l'inverse, les notes après une garde de filière médico-chirurgicale sont plus hautes. Ces différences entre types de garde (SMUR et filière médico-chirurgicale) peuvent s'expliquer par un flux de patients toujours plus intense aux urgences. Le nombre de passages aux urgences a augmenté de $4 \%$ en 2014 par rapport aux flux observés en 2013, indique une étude de la DREES (direction de la recherche, des études, de l'évaluation et des statistiques) dans l'édition 2016 de son panorama des établissements de santé [2]. À Nîmes, entre 2013 et 2014, le nombre de passages a augmenté de $11 \%$. La majoration des flux entraine une augmentation de la violence physique ou verbale [28]. Il pourrait s'agir d'un facteur agissant sur le syndrome PGN. Dans notre étude, le syndrome PGN n'est pas lié à la longueur de la garde (24 vs 12 h) ni au 
nombre de gardes dans le mois. Aux États-Unis, un système en 10/14 h est préconisé pour prévenir les effets de la restriction de sommeil. Pour justifier ces choix, Gaba et al. montre l'incompatibilité entre le manque de sommeil et un système de santé sûr et de qualité [29]. En France, depuis le 10 juillet 2015, la loi définit le temps de travail des urgentistes. Il n'est cependant pas précisé si les $39 \mathrm{~h}$ par semaine doivent être faites sur un modèle $10 / 14 \mathrm{~h}$ ou $24 \mathrm{~h}$ [30].

D'autres recherches de plus grande ampleur pourraient compléter cette démarche initiale (étude du ressenti de l'entourage du médecin urgentiste, conséquences neuroendocrines, cardiovasculaires). Une méthodologie qualitative, un appariement des cas (en post-garde) à leur propre témoin (évaluation à distance) seraient des axes de travail également intéressants. Aussi, une comparaison des symptômes en post-garde immédiat et en journée hors-garde permettrait une description plus fiable méthodologiquement. Il a été montré par Dutheil $\mathrm{F}$ et al. que les conséquences physiques de la gardes pouvaient perdurer jusqu'à trois jours après la garde. Des études complémentaires et plus longues seraient des projets de travail intéressants [31]. Ce travail pourrait être la base de discussions visant à améliorer les conséquences liées à la privation de sommeil.

\section{Conclusion}

Le syndrome PGN se caractérise par des troubles somatiques, des troubles comportementaux, des troubles de l'humeur et des symptômes psychiques. Ces symptômes, quels qu'ils soient, sont moins importants en post-garde immédiat que lorsqu'évalués à distance. Les gardes SMUR et en traumatologie sont mieux tolérées que les gardes de filière médico-chirurgicale. Les discussions pour limiter les conséquences liées à la privation de sommeil et ainsi diminuer les syndromes d'épuisement professionnel peuvent se baser sur cette recherche. Cette démarche pourrait être poursuivie par l'étude du ressenti de l'entourage du médecin urgentiste ou des conséquences neuroendocrines, cardiovasculaires du syndrome PGN.

Conflits d'intérêts : les auteurs déclarent ne pas avoir de liens d'intérêts.

\section{Références}

1. Sand M, Hessam S, Sand D, et al (2016) Stress-coping styles of 459 emergency care physicians in Germany: a pilot study. Anaesthesist 65:841-6

2. Doppia MA, Estryn-Béhar M, Fry C, et al (2011) Burnout in French doctors: a comparative study among anaesthesiologists and other specialists in French hospitals (SESMAT study). Ann Fr Anesth Reanim 30:782-94
3. Regehr C, LeBlanc VR (2017) PTSD, acute stress, performance and decision-making in emergency service workers. J Am Acad Psychiatry Law 45:184-92

4. Ziebertz CM, van Hooff MLM, Beckers DGJ, et al (2015) The relationship of on-call work with fatigue, work-home interference, and perceived performance difficulties. BioMed Res Int 2015:643413

5. Krebs H (2016) La reconversion des médecins urgentistes vers la médecine générale dans le Languedoc-Roussillon : une étude qualitative. http://santenews.reseauprosante.fr/actualite/la-reconversion-des-medecins-urgentistes-vers-la-medecine-generaledans-le-languedoc-roussillon-une-etude-qualitative/344938 (Dernier accès le 18 septembre 2017)

6. Estryn-Behar M, Doppia MA, Guetarni K, et al (2011) Emergency physicians accumulate more stress factors than other physicians-results from the French SESMAT study. Emerg Med J 28:397-410

7. Valatx JL (1998) La privation du sommeil. https://sommeil.univlyon1.fr/articles/valatx/mh_88/print.php. (Dernier accès le 3 décembre 2016)

8. Moser C (2013) Le syndrome postgarde... http://vismaviedejeuneurgentiste.tumblr.com/post/56785730261/le-syndrome-post-garde (Dernier accès le 14 janvier 2017)

9. Faye-Dumanget C, Carré J, Le Borgne M, Boudoukha PAH (2017) French validation of the Maslach Burnout InventoryStudent Survey. J Eval Clin Pract 23:1247-51

10. Voutilainen A, Pitkäaho T, Kvist T, Vehviläinen-Julkunen K (2016) How to ask about patient satisfaction? The visual analogue scale is less vulnerable to confounding factors and ceiling effect than a symmetric Like. J Adv Nurs 72:946-57

11. Fido A, Ghali A (2008) Detrimental effects of variable work shifts on quality of sleep, general health and work performance. Med Princ Pract 17:453-7

12. Hamui-Sutton L, Barragán-Pérez V, Fuentes-García R, et al (2013) Sleep deprivation effects on cognitive, psychomotor skills and its relationship with personal characteristics of resident doctors. Cir Cir 81:317-27

13. Kiernan M, Civetta J, Bartus C, et al (2006) 24 hours on-call and acute fatigue no longer worsen resident mood under the 80-hour work week regulations. Curr Surg 63:237-41

14. Saxena AD, George CFP (2005) Sleep and motor performance in on-call internal medicine residents. Sleep 28:1386-91

15. Ahmed N, Devitt KS, Keshet I, et al (2014) A systematic review of the effects of resident duty hour restrictions in surgery: impact on resident wellness, training, and patient outcomes. Ann Surg 259:1041-53

16. Maric A, Montvai E, Werth E, et al (2017) Insufficient sleep: enhanced risk-seeking relates to low local sleep intensity. Ann Neurol 82:409-18

17. Basner M, Rao H, Goel N, Dinges DF (2013) Sleep deprivation and neurobehavioral dynamics. Curr Opin Neurobiol 23:854-63

18. Banks S, Dinges DF (2017) Behavioral and physiological consequences of sleep restriction. J Clin Sleep Med 3:519-28

19. Krause AJ, Simon EB, Mander BA, et al (2017) The sleepdeprived human brain. Nat Rev Neurosci 18:404-18

20. Knutsson A, Nilsson T (1998) Tobacco use and exposure to environmental tobacco smoke in relation to certain work characteristics. Scand J Soc Med 26:183-9

21. Ker K, Edwards PJ, Felix LM, et al (2010) Caffeine for the prevention of injuries and errors in shift workers. Cochrane Database Syst Rev 12:CD008508

22. Killgore WDS, Kamimori GH, Balkin TJ (2011) Caffeine protects against increased risk-taking propensity during severe sleep deprivation. J Sleep Res 20:395-403 
23. Pecotić R, Valić M, Kardum G, et al (2008) Sleep habits of medical students, physicians and nurses regarding age, sex, shift work and caffein consumption. Lijec Vjesn 130:87-91

24. Smith MR, Eastman CI (2012) Shift work: health, performance and safety problems, traditional countermeasures, and innovative management strategies to reduce circadian misalignment. Nat Sci Sleep 4:111-32

25. Daanen HAM, van Ling S, Tan TK (2013) Subjective ratings and performance in the heat and after sleep deprivation. Aviat Space Environ Med 84:701-7

26. Reid K, Dawson D (2001) Comparing performance on a simulated 12 hour shift rotation in young and older subjects. Occup Environ Med 58:58-62

27. Bae MJ, Song YM, Shin JY, et al (2017) The association between shift work and health behavior: findings from the Korean National
Health and Nutrition Examination Survey. Korean J Fam Med 38:86-92

28. Duchateau FX, Bajolet-Laplante MF, Chollet C, et al (2002) Exposure of French emergency medical personnel to violence. Ann Fr Anesth Reanim 21:775-8

29. Gaba DM, Howard SK (2002) Fatigue among clinicians and the safety of patients. N Engl J Med 347:1249-55

30. Fédération Hospitalière de France (2015) Temps de travail des urgentistes : instruction du 10 juillet 2015. https://www.fhf.fr/Ressources-humaines/Gestion-du-personnel-medical/Temps-de-travaildes-urgentistes-instruction-du-10-juillet-2015 (Dernier accès le 26 août 2017)

31. Dutheil F, Perrie C, Lac G, et al (2013) Urinary Interleukin-8 is a biomarker of stress in emergency physicians, especially with advancing age. PLoS One 8:e71658 